Post-infection platform therapy for mitigating severe cases of COVID-19 infections

\title{
The clinically validated viral superinfection therapy (SIT) platform technology may mitigate severe cases of COVID-19 infections
}

Imre Kovesdi, PhD, ${ }^{1}$, Marc Van Ranst, PhD, ${ }^{2}$, Peter M. Chumakov, ${ }^{3}{ }^{4} \mathrm{MD}, \mathrm{PhD}$, DSc, Volker Sandig, PhD, 5 and Tibor Bakacs, MD, PhD, DSc, ${ }^{*}$

${ }^{1}$ DNAtrix Inc, 2450 Holcombe Blvd. Suite X+ 200, Houston, TX 77021, USA; Tel: 1-301-963-1641, Email: imkovesdi@gmail.com;

2 Laboratory of Clinical Virology, Rega Institute, Herestraat 49 box 1040, 3000 Leuven, Belgium; Tel: +32-16-347 908, Email: vanranstmarc@gmail.com;

3 Engelhardt Institute of Molecular Biology, Russian Academy of Sciences; 32, Vaviolva str., Moscow, 119991, Russia; Tel: +7+495+1352311; e-mail: chumakovpm@yahoo.com; peterchumakov@gmail.com;

${ }^{4}$ Chumakov Federal Scientific Center for Research and Development of Immune and Biological Products, Russian Academy of Sciences, Moscow, Russia; 108819, Российская Федерация, город Москва, поселение Московский, посёлок Института полиомиелита, домовладение 8, корпус 1;

${ }^{5}$ ProBioGen AG, Standort HBS Herbert-Bayer-Straße 8, 13086 Berlin, Germany; Tel: +49 30 322935 - 300; e-mail: Volker.Sandig@probiogen.de

${ }^{6}$ HepC, Inc, 1012 Budapest, Miko str. 3. II. fl. 11., Hungary

(http://www.superinfectiontherapy.com/); Tel: +36-30-726-5122, Email:

tibakacs@hepcinc.com; tiborbakacs@gmail.com;

* Corresponding author: Tibor Bakacs, MD, PhD, DSc

ABSTRACT

The transmission characteristic of COVID-19 is of similar magnitude to severe acute respiratory syndrome-related coronavirus (SARS-CoV) and the 1918 pandemic influenza. The virus is now in more than 100 countries and on nearly all continents. The World Health Organization (WHO) declared the COVID-19 outbreak a pandemic. There is no current evidence from random clinical trials (RCTs) to recommend any specific anti-COVID-19 treatment for patients with suspected or confirmed COVID-19 infection. In order to mitigate the impact of the COVID-19 outbreak, here we propose an innovative superinfection therapeutic (SIT) strategy, which could complement the development of prophylactic vaccines. SIT is based on clinical observations that unrelated viruses might interact in co-infected patients. During SIT, the patient benefit from superinfection with an apathogenic dsRNA virus such as the infectious bursal disease virus (IBDV), which is a powerful activator of the interferon-dependent antiviral gene program. An attenuated vaccine strain of IBDV was already successfully administered to resolve acute and persistent infections induced by two completely different viruses, the hepatitis B (DNA) and C (RNA) viruses (HBV/HCV). Importantly, the epidemiological efficacy of a similar strategy to SIT had already been successfully tested in large controlled trials. Standard live orally administered enterovirus vaccines that stimulate the production of endogenous interferon of the host 
Post-infection platform therapy for mitigating severe cases of COVID-19 infections

mitigated the seasonal outbreaks of influenza and other associated acute respiratory infections in 152,042 individuals without adverse reactions.

Keywords: new coronavirus; COVID-19; superinfection therapy (SIT); apathogenic dsRNA virus; interferon-dependent antiviral genes; broad-spectrum antiviral treatment; clinically tested.

\section{Introduction}

Li et al proposed in the Journal of Medical Virology that the star-like signal and topology of COVID-19 may be indicative of potentially large 'first generation' human-to-human virus transmission (Li, et al., 2020). Riou and Althaus performed stochastic simulations of early outbreak trajectories and found the basic reproduction number (R0) to be around 2.2, indicating the potential for sustained human-to-human transmission (Riou and Althaus, 2020). The transmission characteristics seems to be of similar magnitude as severe acute respiratory syndrome-related coronavirus (SARS-CoV) in 2003 and the 1918 pandemic influenza epidemic. The World Health Organization (WHO) declared SARS-CoV-2 a pandemic. Researchers should therefore move rapidly toward development of medical countermeasures (Phelan, et al., 2020). In order to mitigate the impact of the COVID-19 outbreak, here we propose an innovative therapeutic strategy, which could complement the development of prophylactic vaccines (Kovesdi and Bakacs, 2019) (Bakacs, et al., 2018). The clinically validated viral superinfection therapy (SIT) platform technology may contribute to our pandemic preparedness. SIT is an entirely different modality from the antiviral drugs currently in pipelines as it exploits an apathogenic dsRNA viral vector (infectious bursal diseases virus; IBDV R903/78) that activates the interferon-dependent antiviral gene program of the human body. IFNs are the most potent innate protection when we are immunologically naïve and this response is declining rapidly in aged people (Agrawal, 2013). It has been shown very recently that in contrast to SARS-CoV, SARS-CoV-2 is extremely sensitive to type I IFNs. In Vero cells, IFN alpha treatment reduces SARS-CoV-2 titers by 3-4 orders of magnitude while SARS-CoV replication is reduced only 5-10 fold (Lokugamage, et al., 2020) which makes IBDV R903/78`s interferon activation properties promising to counter SARS-CoV-2 infection. SIT is a therapeutic approach where a nonpathogenic virus is used to infect patients harboring a pathogenic virus and so reduce or eliminate the pathogenic virus caused symptoms. An attenuated vaccine strain of IBDV was already successfully administered to resolve infections induced by two completely different viruses, the hepatitis $B$ (DNA) and C (RNA) viruses (HBV/HCV) in acute and decompensated chronic hepatitis patients (Csatary, et al., 1998) (Csatary, et al., 1999). Importantly, this virus is also a potential vaccine vector drug candidate, since a recombinant IBDV was previously generated that displays exogenous viral peptides from a replication competent IBDV (Upadhyay, et al., 2011). The epidemiological efficacy of a similar strategy to SIT using attenuated vaccine viruses that stimulate the production of endogenous interferon of the host had already been tested in pivotal large scale clinical trials ${ }^{1}$ as described below.

Oral administration of live enterovirus vaccines safely reduced disease incidence in 152,042 healthy individuals during autumn-winter outbreaks of acute respiratory diseases

\footnotetext{
${ }^{1}$ https://www.ncbi.nlm.nih.gov/pubmed/1338742
} 
Post-infection platform therapy for mitigating severe cases of COVID-19 infections

At least 110 types of viruses are now being associated with acute respiratory infections (ARIs). A completely new principle of prevention is required during influenza and other associated acute respiratory infections outbreaks of diseases that come along with actual flu. This principle is based on the use of standard live enterovirus vaccines that stimulate the production of endogenous interferon of the host. To this end, controlled trials of epidemiological efficacy were carried out during three seasonal outbreaks of influenza and other associated acute respiratory infections (between 1968-1971) in 16 regions of three republics in the former Soviet Union. ${ }^{2}$ The surveillance covered about 320,000 people, 2/3 of whom have orally received live enteroviral interferon-inducing vaccine strains (LEV) 2-3 times. No adverse reactions was observed following the administration of LEVs. The enterovirus vaccines provided protection for at least 22,700 people from influenza and ARIs in 152,042 individuals by reducing the incidence on average by 3.2 fold compared to controls who did not receive LEVs. Administration of enterovirus vaccines 2-3 times at intervals of 10-14 days provided 3-5 weeks protection against influenza and acute respiratory infections. Oral administration (in liquid form, or as jelly beans) of the interferon-inducing enterovirus vaccines was the most convenient, but intranasally delivery was also effective. Typically, a dose of vaccine contained 1 million culture infectious units of the virus. The post-infection administration of standard LEVs at the beginning of outbreaks of influenza and ARIs had a therapeutic effect ameliorating the disease.

Importantly, in 12 patients the symptoms of the disease were abolished within 10-12 hours after taking the vaccine. In 6 individuals a significant improvement was observed within 10-12 hours, while the symptoms completely disappeared within 24 hours.

\section{A testable hypothesis}

No specific coronavirus antivirals or vaccines of proven efficacy exist for humans (Wang, et al., 2020). Therefore, in cases of Acute Respiratory Distress Syndrome (ARDS) we propose the use of SIT but only in the context of the Monitored Emergency Use of Unregistered Interventions Framework (MEURI) with strict monitoring. We expect that morbidity and mortality rates of ARDS cases could be substantially reduced by the clinically tested innovative post-exposure SIT. Since interferon is active against most vertebrate-infecting viruses, SIT could be developed into the first biologic drug candidate, for a new broad-spectrum antiviral "one drug, multiple bugs" treatment approach of viral diseases the Science called for (Bekerman and Einav, 2015).

Actually, SIT is a technological platform, which is clearly different from existing approaches that could be scaled up rapidly at low-cost with a strong likelihood of achieving a substantial impact in the near term. Therefore, SIT could be quickly developed into an easy-to-produce and nasally or orally administered therapeutic vaccine to alleviate the deadly lag phase of surge capacity in vaccine production in a pandemic. It is simple to administer and feasible even in resource limited settings.

Even if COVID-19 vaccine production is conducted at breakneck pace as it was announced by the Coalition for Epidemic Preparedness Innovations (CEPI), in the best-case scenario, vaccine will be ready for larger, real-world efficacy tests in humans by summer. ${ }^{3}$ But even if

\footnotetext{
${ }^{2}$ https://www.ncbi.nlm.nih.gov/pubmed/1338742

3 https://www.sciencemag.org/news/2020/01/scientists-are-moving-record-speed-create-new-coronavirus-vaccinesthey-may-come-too
} 
Post-infection platform therapy for mitigating severe cases of COVID-19 infections

experimental vaccines work in clinical trials, mass production is still a huge challenge, which could require year(s).

Because of its major economic importance to the world's poultry industries, attenuated IBDV strains are used as commercial vaccines. These vaccines have an excellent safety record. Despite its worldwide distribution, IBDV is not known to be a hazard in transmitting to any other species than domestic fowl. Furthermore, the vaccine strain of our IBDV drug candidate (R903/78) is harmless in chickens as well.

The clinically tested IBDV-SIT technology platform can be leveraged for the safe, effective, widely affordable post-infection treatment of severe COVID-19 cases

The first Phase 1B/2 clinical trial was performed with a conventionally produced IBDV. Presently, a new Phase 1 trial is planed using a well-characterized drug candidate, which is constructed by reverse genetics and can be manufactured reproducibly. Our preclinical data indicates that R903/78 is a potent activator of the interferon-dependent innate antiviral gene program which could explain its strong interference with unrelated viruses (Hornyak, et al., 2015).

Importantly, IBDV is one of the most stable viruses known that can survive significant temperature variations, which allows for transportation and storage without dry ice. It can endure extreme acidic environments permitting oral delivery. These characteristics would alleviate undeniable logistical challenges of therapeutic vaccine production, transport and cost. Since infections of COVID-19 in China are increasing by the day while the virus is racing around the world, our primary goal is to leverage our clinically tested IBDV-SIT technology platform for the safe, effective, widely affordable post-infection treatment of severe COVID-19 patients, even in resource limited settings. Our major goal is, therefore, to provide the R903/78 drug substance for monitored emergency use in COVID-19 patients suffering from severe illness, including acute respiratory distress syndrome (ARDS) for evaluation. We believe that the R903/78 drug substance could be produced for use in severe COVID-19 patients within six months for evaluation.

Testing the R903/78 drug candidate in severe COVID-19 patients within the Monitored Emergency Use of Unregistered Interventions Framework (MEURI)

To address safety concerns a tissue culture adapted IBDV vaccine strain V903/78 was cloned into cDNA plasmids ensuring easy reproducible production of a reverse engineered virus R903/78. The therapeutic drug candidate was extensively characterized by immunocytochemistry assay (ICC), virus particle determination, and immunoblot analysis. The biodistribution and immunogenicity of the IBDV agent was determined in mice, which is not a natural host of this virus, by quantitative detection of IBDV RNA with qRT-PCR and virus neutralization test (VNT), respectively. The virus was stable at a wide $\mathrm{pH}$ range, demonstrating significant resistance to low $\mathrm{pH}$ and also proved to be highly resistant to high temperatures. No pathological effects were observed in mice and rats, respectively. Single and multiple oral administration of IBDV elicited a neutralizing antibody response that peaked around 21 days post-infection. Repeat oral administration of R903/78 was successful despite the presence of neutralizing antibodies. Viral replication could not be demonstrated in mice and rats, but the powerful induction of IFN related genes was observed. Several human cell lines supported IBDV 
Post-infection platform therapy for mitigating severe cases of COVID-19 infections

propagation in the absence of visible cytopathic effect (CPE) and was secreted into the medium. This resulted in the development of a simple downstream manufacturing process. Several lots of agent were manufactured under research and GLP conditions. Also, different formulations were tested for stability and a formulation that was stable at $5^{\circ} \mathrm{C}$ for 6 months was developed. These preclinical data support the development of an orally or nasally delivered anti-viral agent for human use (Hornyak, et al., 2015).

We wish to develop a nasally or orally administered attenuated IBDV drug candidate (R903/78) for the treatment of severe COVID-19 patients. Initially we will manufacture R903/78 under cGMP conditions to provide sufficient quantity to allow evaluation of 100 patients. Following a successful clinical study, we will manufacture in sufficiently large scale to provide therapy for thousands of patients. It is imperative to develop a next-generation post-infection technology, the SIT platform that can counter increasing threats of the COVID-19 epidemic. We propose therefore an accelerated program to manufacture sufficient experimental R903/78 drug substance to treat approximately 100 patients for 1 month with 1 vial/day/1x10 infectious units of virus (3000 vials). This effort would take about 6 months depending on the release testing required and allowed by EMA and/or FDA. The vials would be shipped to severe COVID19 patients who were admitted to ICU to evaluate the percent of patient recovery from COVID19.

\section{Simplifying the manufacturing process to achieve higher yields at lower costs}

Simplification of the manufacturing processes and the achievement of higher yields could rapidly increase production to provide drug substance in larger quantities. To this end, previous productions in iCELLis bioreactor using Vero cells will be changed to production in serum free and chemically defined media using the Wave bioreactor and ProBioGen's AGE1.CR cell line family. AGE1.CR and AGE1.CR.PIX cells proliferate in suspension in commercially available, chemically defined medium free of animal-derived components and allows robust and fullyscalable proliferation in different bioreactor systems. AGE1.CR cell line family is designed to meet health regulatory guidelines and the 'defined risk' concept issued by the FDA for generation of new cell substrates for application in the production of live vaccines because the immortalizing genes are known and traceable (Jordan, et al., 2009). It was demonstrated that both cell lines AGE1.CR and AGE1.CR.PIX are fully permissive for IBDV strain R903/78. Titers up to $10^{10} \mathrm{TCID}_{50} / \mathrm{ml}$ can be achieved in a 4-day process, more than 1000 times compared to in VERO cells, which secures a good yield for a commercially viable virus production.

Incorporation of COVID-19 specific epitopes into the IBDV capsid to provide coronavirus specific immune responses

We wish to improve the efficacy of the R903/78 IBDV vector by incorporating disease specific epitopes. Since it was already demonstrated that SIT generates strong anti-IBDV antibody response, it is our hypotheses that incorporation of COVID-19 specific epitopes into the IBDV capsid will be able to provide coronavirus specific immune responses, which will synergize with the SIT technology. Therefore, we will incorporate the genetic sequence for COVID-19's spike epitopes into the R903/78 vector and recombinant IBDV vectors carrying COVID-19 spike epitopes will be generated (Upadhyay, et al., 2011). These vectors will be evaluated for their ability to generate immune responses against COVID-19. Vectors with the most promising 
Post-infection platform therapy for mitigating severe cases of COVID-19 infections

characteristics will be provided to collaborators to be tested for their protection against a lethal challenge in appropriate animal models.

Extending our knowledge on virus induced changes and mechanisms of action of SIT by developing animal models for severe virus infections

The development of animal model(s) for better understanding of the mechanisms of action for COVID-19 and other serious viral diseases is planned. It was demonstrated that SARS-CoV can infect the kidney cells of the African green monkey (Vero E6) (Li, et al., 2003). The Institute Pasteur recently announced that they successfully cultured the samples from COVID-19 positive patients on Vero E6 cells. ${ }^{4}$ While generating small animal models of coronavirus disease can be difficult, SARS-CoV passaged to adapt to the mouse host caused significant disease in mice (Frieman, et al., 2012) (Gralinski and Menachery, 2020).

In our mice studies (Hornyak, et al., 2015), we demonstrated that using high doses of R903/78 inoculums repeatedly is feasible. Therefore, we hope to demonstrate that breakthrough infection and sustained viremia can be achieved and maintained in small animal models, in spite of the presence of anti-IBDV antibodies. We have preliminary evidence that the doublestranded RNA drug candidate virus (R903/78) switches on some of the major activators of the interferon-dependent innate antiviral gene program. Expression levels of IRF-7, IRF3, TLR3 and 9, ZBP1, Ifi204 genes significantly increased (e.g. IRF7 up to 267 times over base line) in the liver tissue following a single administration of R903/78 in mice (unpublished observation). Importantly, long-term administration of large doses (400 times that of planed therapeutic dose) of the R903/78 drug candidate showed no toxic effects in mice and rats. Therefore, key activators of the interferon-dependent innate antiviral gene program will be measured. The expression levels of genes over time in organs and tissues of animals during nasal and oral administration of the R903/78 drug candidate will be determined. Cytokine release will be determined at different stages of R903/78 virus infection during the exacerbated innate immune response. In addition, antibody titers will be measured in animals during multiple nasal and oral administration.

\section{Conclusion}

The COVID-19 epidemic is declared a public health emergency of international concern (PHEIC). It is imperative to develop a next-generation post-infection technology, the SIT platform that can counter increasing threats of the COVID-19 epidemic. We propose therefore the testing of the R903/78 drug candidate in severe COVID-19 patients within the Monitored Emergency Use of Unregistered Interventions Framework (MEURI). We propose an accelerated program to manufacture sufficient experimental R903/78 drug substance to treat approximately 100 patients for 1 month with 1 vial/day/1x10 infectious units of virus (3000 vials). This effort would take about 6 months depending on the release testing required and allowed by EMA and/or FDA. The vials would be shipped to severe COVID-19 patients who were admitted to ICU to evaluate the percent of patient recovery from COVID-19.

\footnotetext{
${ }^{4}$ https://www.pasteur.fr/en/press-area/press-documents/institut-pasteur-isolates-strains-coronavirus-2019-ncovdetected-france
} 
Post-infection platform therapy for mitigating severe cases of COVID-19 infections

Conflict of Interest: I.K., M.V.R. and T.B. are shareholders of HepC, Inc., V.S. is listed as an inventor on patents that protects the AGE1.CR cell line family (WO 2005/042728 and WO2007/054516)

\section{References}

Li, X., Zai, J., Wang, X., Li, Y. 2020. Potential of large 'first generation' human-to-human transmission of 2019-nCoV. J Med Virol.

Riou, J., Althaus, C.L. 2020. Pattern of early human-to-human transmission of Wuhan 2019nCoV. bioRxiv, 2020.01.23.917351.

Phelan, A.L., Katz, R., Gostin, L.O. 2020. The Novel Coronavirus Originating in Wuhan, China: Challenges for Global Health Governance. JAMA.

Kovesdi, I., Bakacs, T. 2019. Therapeutic exploitation of viral interference. Infectious Disorders Drug Targets 19, 1.

Bakacs, T., Safadi, R., Kovesdi, I. 2018. Post-infection viral superinfection technology could treat HBV and HCV patients with unmet needs. Hepatol Med Policy 3, 2.

Agrawal, A. 2013. Mechanisms and implications of age-associated impaired innate interferon secretion by dendritic cells: a mini-review. Gerontology 59, 421.

Lokugamage, K.G., Schindewolf, C., Menachery, V.D. 2020. SARS-CoV-2 sensitive to type I interferon pretreatment. bioRxiv, 2020.03.07.982264.

Csatary, L.K., Telegdy, L., Gergely, P., Bodey, B., Bakacs, T. 1998. Preliminary report of a controlled trial of MTH-68/B virus vaccine treatment in acute $B$ and $C$ hepatitis: a phase II study. Anticancer Res 18, 1279.

Csatary, L.K., Schnabel, R., Bakacs, T. 1999. Successful treatment of decompensated chronic viral hepatitis by bursal disease virus vaccine. Anticancer Res 19, 629.

Upadhyay, C., Ammayappan, A., Patel, D., Kovesdi, I., Vakharia, V.N. 2011. Recombinant infectious bursal disease virus carrying hepatitis C virus epitopes. J Virol 85, 1408.

Wang, C., Horby, P.W., Hayden, F.G., Gao, G.F. 2020. A novel coronavirus outbreak of global health concern. The Lancet.

Bekerman, E., Einav, S. 2015. Infectious disease. Combating emerging viral threats. Science 348, 282.

Hornyak, A., Lipinski, K.S., Bakonyi, T., Forgach, P., Horvath, E., Farsang, A., Hedley, S.J., Palya, V., Bakacs, T., Kovesdi, I. 2015. Effective multiple oral administration of reverse genetics engineered infectious bursal disease virus in mice in the presence of neutralizing antibodies. J Gene Med 17, 116.

Jordan, I., Vos, A., Beilfuss, S., Neubert, A., Breul, S., Sandig, V. 2009. An avian cell line designed for production of highly attenuated viruses. Vaccine 27, 748.

Li, W., Moore, M.J., Vasilieva, N., Sui, J., Wong, S.K., Berne, M.A., Somasundaran, M., Sullivan, J.L., Luzuriaga, K., Greenough, T.C., Choe, H., Farzan, M. 2003. Angiotensin-converting enzyme 2 is a functional receptor for the SARS coronavirus. Nature 426, 450.

Frieman, M., Yount, B., Agnihothram, S., Page, C., Donaldson, E., Roberts, A., Vogel, L., Woodruff, B., Scorpio, D., Subbarao, K., Baric, R.S. 2012. Molecular determinants of severe acute respiratory syndrome coronavirus pathogenesis and virulence in young and aged mouse models of human disease. J Virol 86, 884. 
Post-infection platform therapy for mitigating severe cases of COVID-19 infections

Gralinski, L.E., Menachery, V.D. 2020. Return of the Coronavirus: 2019-nCoV. Viruses 12. 